\title{
Green chemistry approach to synthesis of some new trifluoromethyl containing tetrahydropyrimidines under solvent free conditions
}

\author{
R. C. Khunt, J. D. Akbari, A. T. Manvar, S. D. Tala, M. F. Dhaduk, \\ H. S. Joshi and Anamik Shah* \\ Department of Chemistry, Saurashtra University, Rajkot-360005, Gujarat, India \\ E-mail:anamik_shah@hotmail.com,drhsjoshi49@gmail.com
}

\begin{abstract}
A simple, efficient and modified Biginelli procedure was carried out for the synthesis of tetrahydropyrimidines $\mathbf{4 a - 0}$ by a solvent-free and catalyst-free condition, by the condensation of 1,3-dicarbonyl compound $\mathbf{1}$, arylaldehydes $\mathbf{2}$ and urea/thiourea $\mathbf{3}$. Neat reactants subjected to microwave irradiation gave the required products more quickly and in better yield in comparison to traditional methodologies. The observed yields and improvement in reaction rates are due to the solvent free conditions coupled with the use of microwave radiation.
\end{abstract}

Keywords: Tetrahydropyrimidines, microwave irradiation synthesis, solvent-free and catalystfree

\section{Introduction}

Environmental concerns in research and industry are increasing ${ }^{1}$ with the increasing pressure to reduce the amount of pollutants produced, including organic solvents whose recovery is mandated by ever more strict laws. Hence the challenge for a sustainable environment calls for the use of clean procedures to avoid the use of harmful solvents. The emergence of microwave assisted solid phase synthesis ${ }^{2}$ is a step forward in this direction. In this expeditious and solventfree approach ${ }^{3}$ the adsorbed reactants over solid supports are exposed to microwave irradiation. The salient features of these high yield protocols with enhanced reaction rates are greater selectivity and experimental ease of manipulation, ${ }^{4}$ but this technique does not exactly meet the definition of 'no solvent'. The usage of solvent is only eliminated at the primary reaction stage whereas an appreciable amount of solvent is still required for the adsorption of reactants and elution of the product at the pre- and post- reaction stages, respectively. A "neat reaction" is an alternative solvent-free approach that eliminates the use of a solid support as well as solvent from the reaction. There has not been much advancement in this area as direct heating of the reactants in the absence of solvent with a solid support often leads to charring. But these 
reactions can prove to be advantageous for environmental reasons and can also offer the benefits of shorter reaction times especially, when coupled with microwave radiation ${ }^{6}$ due to their uniform heating effect.

Dihydropyrimidines and their derivatives ${ }^{7}$ are medicinally important ${ }^{8}$ as calcium channel blockers, antihypertensive and anti-inflammatory agents and $\alpha_{1 \mathrm{a}}$-antagonists. The first one-pot synthesis of 3,4-dihydropyrimidine was reported by Biginelli ${ }^{9}$ in 1893. A serious drawback of the original procedure was low yield with substituted aliphatic and aromatic aldehydes. ${ }^{10}$ Several improved procedures have been reported using Lewis acids catalysts such as $\mathrm{BF}_{3},{ }^{11 a} \mathrm{FeCl}_{3},{ }^{11 b}$ $\mathrm{InCl}_{3},{ }^{11 c} \mathrm{BiCl}_{3},{ }^{11 d} \mathrm{LaCl}_{3},{ }^{11 e} \mathrm{LiClO}_{4},{ }^{11 f} \mathrm{Mn}-(\mathrm{OAc})_{3},{ }^{11 g} \mathrm{CAN},{ }^{11 h}$ in a solvent such as $\mathrm{CH}_{3} \mathrm{CN}$, $\mathrm{CH}_{2} \mathrm{Cl}_{2}$, or THF. Recently, a number of procedures under solvent-free conditions using $\mathrm{Y}\left(\mathrm{NO}_{3}\right)_{3} \cdot 6 \mathrm{H}_{2} \mathrm{O},{ }^{11 i}$ silica triflate ${ }^{11 j}$ lanthanide triflate ${ }^{11 k}$, samarium diiodide ${ }^{111}$ and ionic liquid ${ }^{11 m}$ as catalysts have also been reported. Obviously, many of these catalysts and solvents are not at all acceptable in the context of green synthesis.

Thus, in the present paper we look forward to green synthesis of the Biginelli reaction (Scheme 1) under solvent free condition. The equimolar amount of neat 1,3-dicarbonyl compound 1, different aromatic aldehydes $\mathbf{2}$ and urea/thiourea $\mathbf{3}$ on the exposure to microwave irradiation, which gave the required products 4a-o without using solid support, solvent or acid. ${ }^{12,13}$ The product was isolated by triturating with distilled water.

In general, the reactions are very clean, without any side product in every run. In fact, the crude products obtained are of high purity $\left(>90 \%\right.$ by ${ }^{1} \mathrm{H}$ NMR) with remarkable yields and do not require any chromatographic separation. Recrystallization from hot ethanol provides analytically pure sample. Most significantly, the whole operation involves no organic solvent at any stage.

\section{Results and Discussions}

The present procedure for the synthesis of tetrahydropyrimidines by a solvent-free and catalystfree condensation of 1,3-dicarbonyl compound, aldehyde, and urea/thiourea provides a simple, efficient, cost-effective with $100 \%$ green modification of the Biginelli's reaction. Most significantly, this solvent-free and catalyst-free process of three-component condensation throws a challenge to the existing procedures ${ }^{11,14}$ which use volatile and hazardous solvents and toxic catalysts, and in general lead to a new direction in organic synthesis.

The 1,3-dicarbonyl i.e. 4,4,4-trifluoro-1-(4-methoxypheny)butane-1,3-dione 1 was cyclized with arylaldehydes $\mathbf{2}$ and urea/thiourea $\mathbf{3}$ to give the tetrahydropyrimidines ${ }^{15} \mathbf{4 a - 0}$ (Scheme 1), which was considered to be a final product in Biginelli reaction. The structure of the final products was confirmed by the earlier report by Saloutin, V. I et al., ${ }^{16}$ Kappe et al. ${ }^{17}$ and D. Subhas Bose et al. ${ }^{18}$ In the ${ }^{1} \mathrm{H}$ NMR spectrum of $\mathbf{4 b}$, the most characteristic signals are two doublets at $\underline{4.14}-4.16$ and $\underline{4.96}-4.99 \mathrm{\delta ppm}$ which are corresponding to the trans-axial methane protons. The observed coupling constant $J=10.96 \mathrm{~Hz}$ and $11.0 \mathrm{~Hz}$ assigned to the $\mathrm{H}_{4}$ and $\mathrm{H}_{5}$ 
protons respectively, agree very well with the values found in the references. ${ }^{16,18}$ It is therefore reasonable to assume that the same relative stereochemistry appears in 4a-o. It may be presumed that the $-\mathrm{OH}$ group at $\mathrm{C}-6$ may be cis to $\mathrm{H}_{5}$, thereby the elimination of water requires drastic conditions. In MS the molecular ion peak appears at $424 \mathrm{~m} / \mathrm{z}$ which was further supports that the elimination of water does not take place.<smiles>COc1ccc(C(=O)CC(=O)C(F)(F)F)cc1</smiles>

1<smiles>[R]C=O</smiles><smiles>CC#N</smiles>

3<smiles>[X]C1=N[C@]([2H])([C@@H](C(=O)c2ccc(OC)cc2)C(F)(F)F)[C@]([2H])(O)N1</smiles>

4a-o

Where $\mathrm{X}=\mathrm{O}, \mathrm{S}$

$\mathrm{R}=$ Aryl

\section{Scheme 1}

Table 1. Synthesis of 1,4-dihydropyrimidine without any solvent and catalyst

\begin{tabular}{|c|c|c|c|c|c|}
\hline Compd. & $\mathrm{R}$ & $X$ & Yield $(\%)_{a}$ & $m p{ }^{\circ} \mathrm{C}$ & Time $/ \mathrm{min}$ \\
\hline $4 \mathbf{a}$ & & $\mathrm{O}$ & 85 & $180-182$ & 1.5 \\
\hline $4 b$ & & $\mathrm{O}$ & 82 & $205-206$ & 2.0 \\
\hline $4 c$ & & $\mathrm{O}$ & 79 & $212-214$ & 1.5 \\
\hline $4 d$ & & $\mathrm{O}$ & 83 & $198-200$ & 4.5 \\
\hline $4 e$ & & $\mathrm{O}$ & 82 & $160-162$ & 6.5 \\
\hline $4 f$ & & $\mathrm{O}$ & 84 & $220-222$ & 3.0 \\
\hline $4 g$ & & $\mathrm{O}$ & 78 & $234-236$ & 3.5 \\
\hline
\end{tabular}




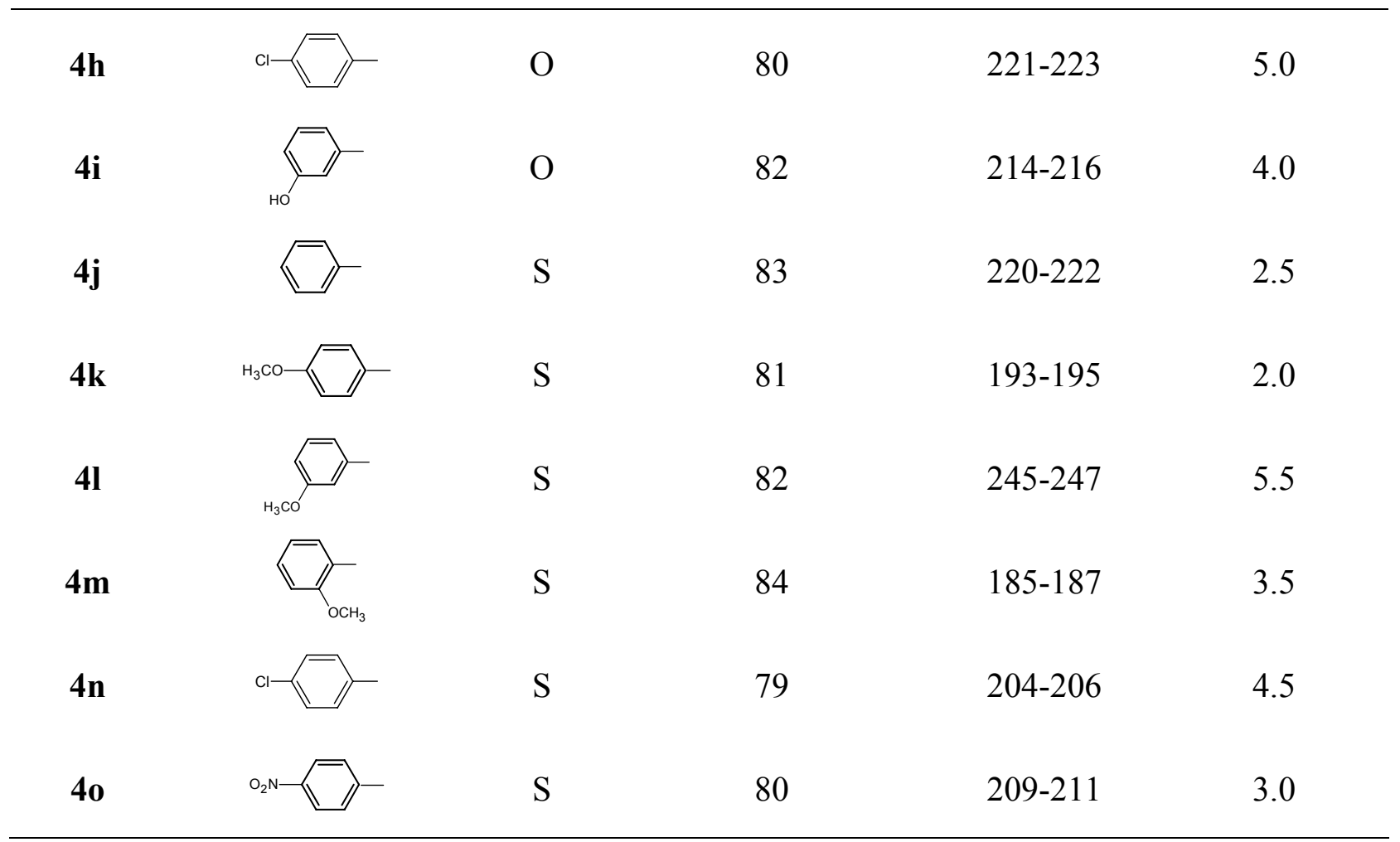

a Yields refer to those of recrystallized pure products characterized by mp and spectral data (IR, ${ }^{1} \mathrm{H}$ NMR and mass spectra).

\section{Experimental Section}

General Procedures. Melting points were measured in open capillaries and are uncorrected. The syntheses were carried out in a Questron Technologies Corp. QPro-M microwave synthesizer. Elemental analyses were performed on a Carlo Erba EA 1108 elemental analyzer at SAIF, CDRI Lucknow. IR spectra were recorded on $\mathrm{KBr}$ discs, using FTIR-8400 spectrophotometer. ${ }^{1} \mathrm{H}$ NMR spectra were taken on a Bruker AVANCE II 400 ( ${ }^{1} \mathrm{H}: 400 \mathrm{Mz}$, [d $\mathrm{d}_{6}$. DMSO) spectrometer. Mass spectra were determined using direct inlet probe on a GCMS-QP2010 mass spectrometer. Analytical thin layer chromatography (TLC) was performed on Silica Gel 60 F254 precoated plates.

\section{General procedure for the preparation of tetrahydropyrimidines}

A mixture of 4,4,4-trifluoro-1-(4-methoxypheny)butane-1,3-dione 1 (5 mmol), different aromatic aldehydes 2 (5 mmol) and thio/urea $3(5 \mathrm{mmol})$ was placed in a flask and irradiated under microwave at the power of $600 \mathrm{~W}$ and $110-120^{\circ} \mathrm{C}$ for different time which is described in Table 1. After cooling, the resulting solid was crushed, washed with cold water, filtered and dried under vacuum to give the crude product which is reasonably pure ( $>90 \%$, purity by $\left.{ }^{1} \mathrm{H} \mathrm{NMR}\right)$. However, recrystallization from hot ethanol provides the analytically pure product. 
4-Hydroxy-5-(4-methoxybenzoyl)-6-phenyl-4-(trifluoromethyl)tetrahydropyrimidin-2(1H)one (4a). White powder in 85\% yield. mp 180-182 ${ }^{\circ} \mathrm{C}$. IR (KBr): $v 3447(-\mathrm{NH}), 3209(-\mathrm{OH})$, $3109(\mathrm{ArH}), 1691$ and $1676(\mathrm{C}=\mathrm{O}) \mathrm{cm}^{-1} .{ }^{1} \mathrm{H}$ NMR (400MHz, DMSO-d 6$): \delta 3.80\left(\mathrm{~s}, 3 \mathrm{H}, \mathrm{OCH}_{3}\right)$, $4.17(\mathrm{~d}, 1 \mathrm{H}, \mathrm{J}=11.0 \mathrm{~Hz}), 5.03(\mathrm{~d}, 1 \mathrm{H}, \mathrm{J}=11.0 \mathrm{~Hz}), 6.49(\mathrm{~s}, 1 \mathrm{H}, \mathrm{NH}), 6.64(\mathrm{~s}, 1 \mathrm{H}, \mathrm{NH}), 6.72-7.54$ (m, 9H, Ar-H). MS m/z: $394\left(\mathrm{M}^{+}\right)$. Anal. Calcd. for $\mathrm{C}_{19} \mathrm{H}_{17} \mathrm{~F}_{3} \mathrm{~N}_{2} \mathrm{O}_{4}: \mathrm{C}, 57.87 ; \mathrm{H}, 4.35, \mathrm{~N}, 7.10 \%$. Found: C, 57.75; H, 4.12, N, 6.93\%.

4-Hydroxy-5-(4-methoxybenzoyl)-6-(4-methoxyphenyl)-4-(trifluoromethyl)tetrahydropyrimidin-2(1H)-one (4b). White solid in 82\% yield. mp 205-206 ${ }^{\circ} \mathrm{C}$. IR (KBr): $v 3443(-\mathrm{NH}), 3217$ (-OH), $3070(\mathrm{ArH}), 1676$ and $1668(\mathrm{C}=\mathrm{O}) \mathrm{cm}^{-1} .{ }^{1} \mathrm{H}$ NMR (400MHz, DMSO- $\left.d_{6}\right): \delta 3.67$ (s, 3H, $\left.\mathrm{OCH}_{3}\right), 3.82\left(\mathrm{~s}, 3 \mathrm{H}, \mathrm{OCH}_{3}\right), 4.16(\mathrm{~d}, 1 \mathrm{H}, \mathrm{J}=11.0 \mathrm{~Hz}), 4.99(\mathrm{~d}, 1 \mathrm{H}, \mathrm{J}=10.9 \mathrm{~Hz}), 6.13(\mathrm{~s}, 1 \mathrm{H}, \mathrm{NH})$, 6.49 (s, 1H, NH), 6.67-6.70 (dd, 2H, Ar-H, J = 6.9Hz), 6.75-6.77 (dd, 2H, Ar-H, J = 7.0Hz), 7.20-7.22 (dd, 2H, Ar-Ha-a' J =6.8Hz), 7.56-7.58 (dd, 2H, Ar-Hb-b' J = 7.0Hz). MS m/z: 424 $\left(\mathrm{M}^{+}\right)$. Anal. Calcd. for $\mathrm{C}_{20} \mathrm{H}_{19} \mathrm{~F}_{3} \mathrm{~N}_{2} \mathrm{O}_{5}$ : C, 56.60; H, 4.51, N, 6.60\%. Found: C, 56.48; H, 4.29, N, $6.25 \%$.

4-Hydroxy-5-(4-methoxybenzoyl)-6-(2-methoxyphenyl)-4-(trifluoromethyl)tetrahydropyrimidin-2(1H)-one (4c). White powder in 79\% yield. mp 212-214 ${ }^{\circ} \mathrm{C}$. IR (KBr): $v 3429(-\mathrm{NH})$, $3205(-\mathrm{OH}), 3101(\mathrm{ArH}), 1687$ and $1676(\mathrm{C}=\mathrm{O}) \mathrm{cm}^{-1} .{ }^{1} \mathrm{H}$ NMR (400MHz, DMSO- $\left.d_{6}\right): \delta 3.67(\mathrm{~s}$, $\left.3 \mathrm{H}, \mathrm{OCH}_{3}\right), 3.81\left(\mathrm{~s}, 3 \mathrm{H}, \mathrm{OCH}_{3}\right), 4.43(\mathrm{~d}, 1 \mathrm{H}, \mathrm{J}=11.0 \mathrm{~Hz}), 5.31(\mathrm{~d}, 1 \mathrm{H}, \mathrm{J}=10.8 \mathrm{~Hz}), 5.93(\mathrm{~s}, 1 \mathrm{H}$, $\mathrm{NH}), 6.43(\mathrm{~s}, 1 \mathrm{H}, \mathrm{NH}), 6.56-7.54(\mathrm{~m}, 8 \mathrm{H}, \mathrm{Ar}-\mathrm{H})$. MS $m / z: 424\left(\mathrm{M}^{+}\right)$. Anal. Calcd. for $\mathrm{C}_{20} \mathrm{H}_{19} \mathrm{~F}_{3} \mathrm{~N}_{2} \mathrm{O}_{5}$ : C, 56.60; H, 4.51, N, 6.60\%. Found: C, 56.47; H, 4.31, N, 6.27\%.

4-Hydroxy-5-(4-methoxybenzoyl)-6-(3,4-methoxyphenyl)-4-(trifluoromethyl)tetrahydropyrimidin-2(1H)-one (4d). White crystal in 83\% yield. mp 198-200 ${ }^{\circ} \mathrm{C}$. IR (KBr): $v 3599(-\mathrm{NH})$, $3215(-\mathrm{OH}), 3076(\mathrm{ArH}), 1691$ and $1670(\mathrm{C}=\mathrm{O}) \mathrm{cm}^{-1} .{ }^{1} \mathrm{H}$ NMR $\left(400 \mathrm{MHz}, \mathrm{DMSO}-d_{6}\right): \delta 3.70(\mathrm{~s}$, $\left.3 \mathrm{H}, \mathrm{OCH}_{3}\right), 3.80\left(\mathrm{~s}, 3 \mathrm{H}, \mathrm{OCH}_{3}\right), 3.81\left(\mathrm{~s}, 3 \mathrm{H}, \mathrm{OCH}_{3}\right), 4.24(\mathrm{~d}, 1 \mathrm{H}, \mathrm{J}=10.9 \mathrm{~Hz}), 4.96(\mathrm{~d}, 1 \mathrm{H}$, $\mathrm{J}=10.9 \mathrm{~Hz}), 6.64-7.73(\mathrm{~m}, 9 \mathrm{H}, \mathrm{Ar}-\mathrm{H})$. MS m/z: $454\left(\mathrm{M}^{+}\right)$. Anal. Calcd. for $\mathrm{C}_{21} \mathrm{H}_{21} \mathrm{~F}_{3} \mathrm{~N}_{2} \mathrm{O}_{6}$ : C, 55.51 ; H, 4.66, N, 6.16\%. Found: C, 55.36; H, 4.37, N, 6.03\%.

4-Hydroxy-5-(4-methoxybenzoyl)-6-(2,5-methoxyphenyl)-4-(trifluoromethyl)tetrahydropyrimidin-2(1H)-one (4e). White crystal in $82 \%$ yield. mp $160-162{ }^{\circ} \mathrm{C}$. IR (KBr): $v 3365(-\mathrm{NH})$, $3205(-\mathrm{OH}), 3085(\mathrm{ArH}), 1680$ and $1599(\mathrm{C}=\mathrm{O}) \mathrm{cm}^{-1} .{ }^{1} \mathrm{H}$ NMR (400MHz, DMSO- $\left.d_{6}\right): \delta 3.61(\mathrm{~s}$, $\left.3 \mathrm{H}, \mathrm{OCH}_{3}\right), 3.69\left(\mathrm{~s}, 3 \mathrm{H}, \mathrm{OCH}_{3}\right), 3.82\left(\mathrm{~s}, 3 \mathrm{H}, \mathrm{OCH}_{3}\right), 4.24(\mathrm{~d}, 1 \mathrm{H}, \mathrm{J}=11.0 \mathrm{~Hz}), 5.27(\mathrm{~d}, 1 \mathrm{H}$, $\mathrm{J}=10.7 \mathrm{~Hz}), 6.34(\mathrm{~s}, 1 \mathrm{H}, \mathrm{NH}), 6.91(\mathrm{~s}, 1 \mathrm{H}, \mathrm{NH}), 6.51-7.61(\mathrm{~m}, 7 \mathrm{H}, \mathrm{Ar}-\mathrm{H}) . \mathrm{MS} m / z: 454\left(\mathrm{M}^{+}\right)$. Anal. Calcd. for $\mathrm{C}_{21} \mathrm{H}_{21} \mathrm{~F}_{3} \mathrm{~N}_{2} \mathrm{O}_{6}$ : C, 55.51; H, 4.66, N, 6.16\%. Found: C, 55.33; H, 4.44, N, $6.00 \%$.

4-Hydroxy-5-(4-methoxybenzoyl)-6-(3-nitroyphenyl)-4-(trifluoromethyl)tetrahydropyrimidin-2(1H)-one (4f). Pale white powder in 84\% yield. mp 220-222 ${ }^{\circ} \mathrm{C}$. IR (KBr): v 3463 (-NH), $3196(-\mathrm{OH}), 3046(\mathrm{ArH}), 1691$ and $1676(\mathrm{C}=\mathrm{O}) \mathrm{cm}^{-1} .{ }^{1} \mathrm{H}$ NMR (400MHz, DMSO-d $)_{6}$ : $\delta 3.80\left(\mathrm{~s}, 3 \mathrm{H}, \mathrm{OCH}_{3}\right), 4.27(\mathrm{~d}, 1 \mathrm{H}, \mathrm{J}=11.0 \mathrm{~Hz}), 5.19(\mathrm{~d}, 1 \mathrm{H}, \mathrm{J}=11.0 \mathrm{~Hz}), 6.94(\mathrm{~s}, 1 \mathrm{H}, \mathrm{NH}), 7.17$ (s, $1 \mathrm{H}, \mathrm{NH}), 6.74-8.24(\mathrm{~m}, 8 \mathrm{H}, \mathrm{Ar}-\mathrm{H})$. MS m/z: $439\left(\mathrm{M}^{+}\right)$. Anal. Calcd. for $\mathrm{C}_{19} \mathrm{H}_{16} \mathrm{~F}_{3} \mathrm{~N}_{3} \mathrm{O}_{6}$ : C, 51.94; H, 3.67, N, 9.56\%. Found: C, 51.83; H, 3.58, N, 9.33\%. 
4-Hydroxy-5-(4-methoxybenzoyl)-6-(4-nitrophenyl)-4-(trifluoromethyl)tetrahydropyrimidin-2(1H)-one (4g). White powder in 78\% yield. mp 234-236 ${ }^{\circ} \mathrm{C}$. IR (KBr): $v 3429(-\mathrm{NH})$, $3234(-\mathrm{OH}), 3107(\mathrm{ArH}), 1710$ and $1689(\mathrm{C}=\mathrm{O}) \mathrm{cm}^{-1} .{ }^{1} \mathrm{H}$ NMR (400MHz, DMSO- $\left.d_{6}\right): \delta 3.80(\mathrm{~s}$, $\left.3 \mathrm{H}, \mathrm{OCH}_{3}\right), 4.24(\mathrm{~d}, 1 \mathrm{H}, \mathrm{J}=11.0 \mathrm{~Hz}), 5.20(\mathrm{~d}, 1 \mathrm{H}, \mathrm{J}=11.0 \mathrm{~Hz}), 7.07(\mathrm{~s}, 1 \mathrm{H}, \mathrm{NH}), 7.20(\mathrm{~s}, 1 \mathrm{H}$, $\mathrm{NH}), 6.76-8.03(\mathrm{~m}, 8 \mathrm{H}, \mathrm{Ar}-\mathrm{H})$. MS m/z: $439\left(\mathrm{M}^{+}\right)$. Anal. Calcd. for $\mathrm{C}_{19} \mathrm{H}_{16} \mathrm{~F}_{3} \mathrm{~N}_{3} \mathrm{O}_{6}: \mathrm{C}, 51.94 ; \mathrm{H}$, 3.67, N, 9.56\%. Found: C, 51.85; H, 3.55, N, 9.36\%.

4-Hydroxy-5-(4-methoxybenzoyl)-6-(4-chlorophenyl)-4-(trifluoromethyl)tetrahydropyrimidin-2(1H)-one (4h). Solid white in 80\% yield. mp 221-223 ${ }^{\circ} \mathrm{C}$. IR (KBr): $v 3456(-\mathrm{NH}), 3212$ (-OH), $3117(\mathrm{ArH}), 1685$ and $1672(\mathrm{C}=\mathrm{O}) \mathrm{cm}^{-1} .{ }^{1} \mathrm{H}$ NMR (400MHz, DMSO- $\left.d_{6}\right): \delta 3.82(\mathrm{~s}, 3 \mathrm{H}$, $\left.\mathrm{OCH}_{3}\right), 4.13(\mathrm{~d}, 1 \mathrm{H}, \mathrm{J}=10.9 \mathrm{~Hz}), 5.02(\mathrm{~d}, 1 \mathrm{H}, \mathrm{J}=10.9 \mathrm{~Hz}), 5.65(\mathrm{~s}, 1 \mathrm{H}, \mathrm{NH}), 6.06(\mathrm{~s}, 1 \mathrm{H}, \mathrm{NH})$, 6.75-7.55 (m, 8H, Ar-H). MS m/z: $428\left(\mathrm{M}^{+}\right)$. Anal. Calcd. for $\mathrm{C}_{19} \mathrm{H}_{16} \mathrm{ClF}_{3} \mathrm{~N}_{2} \mathrm{O}_{4}: \mathrm{C}, 53.22 ; \mathrm{H}$, 3.76 , N, 6.53\%. Found: C, 53.06; H, 3.58, N, 6.31\%.

4-Hydroxy-5-(4-methoxybenzoyl)-6-(3-hydroxyphenyl)-4-(trifluoromethyl)tetrahydropyrimidin-2(1H)-one (4i). Solid white in 82\% yield. mp 214-216 ${ }^{\circ} \mathrm{C}$. IR (KBr): $v 3445(-\mathrm{NH}), 3218$ (-OH), $3087(\mathrm{ArH}), 1710$ and $1682(\mathrm{C}=\mathrm{O}) \mathrm{cm}^{-1} .{ }^{1} \mathrm{H}$ NMR (400MHz, DMSO- $\left.d_{6}\right): \delta 3.76(\mathrm{~s}, 3 \mathrm{H}$, $\left.\mathrm{OCH}_{3}\right), 4.09(\mathrm{~d}, 1 \mathrm{H}, \mathrm{J}=10.7 \mathrm{~Hz}), 4.88(\mathrm{~d}, 1 \mathrm{H}, \mathrm{J}=11.0 \mathrm{~Hz}), 6.20(\mathrm{~s}, 1 \mathrm{H}, \mathrm{NH}), 6.44(\mathrm{~s}, 1 \mathrm{H}, \mathrm{NH})$, 6.69-7.53 (m, 8H, Ar-H), $7.71(\mathrm{~s}, 1 \mathrm{H}, \mathrm{OH}), \mathrm{MS} m / z$ : $410\left(\mathrm{M}^{+}\right)$. Anal. Calcd. for $\mathrm{C}_{19} \mathrm{H}_{17} \mathrm{~F}_{3} \mathrm{~N}_{2} \mathrm{O}_{5}$ : C, 55.61; H, 4.18, N, 6.83\%. Found: C, 55.47; H, 4.06, N, 6.68\%.

4-Hydroxy-5-(4-methoxybenzoyl)-6-phenyl-4-(trifluoromethyl)tetrahydropyrimidin-2(1H)thione (4j). Solid white in 83\% yield. mp 220-222 ${ }^{\circ} \mathrm{C}$. IR (KBr): v $3445(-\mathrm{NH}), 3234(-\mathrm{OH})$, 3132 (ArH), $1674(\mathrm{C}=\mathrm{O}) \mathrm{cm}^{-1} .{ }^{1} \mathrm{H}$ NMR (400MHz, DMSO-d $\left.)_{6}\right): \delta .80\left(\mathrm{~s}, 3 \mathrm{H}, \mathrm{OCH}_{3}\right), 4.22$ $(\mathrm{d}, 1 \mathrm{H}, \mathrm{J}=11.0 \mathrm{~Hz}), 5.05(\mathrm{~d}, 1 \mathrm{H}, \mathrm{J}=11.3 \mathrm{~Hz}), 6.74-7.56(\mathrm{~m}, 9 \mathrm{H}, \mathrm{Ar}-\mathrm{H}), 7.76(\mathrm{~s}, 1 \mathrm{H}, \mathrm{NH}), 8.46$ (s, $1 \mathrm{H}, \mathrm{NH})$. MS m/z: $410\left(\mathrm{M}^{+}\right)$. Anal. Calcd. for $\mathrm{C}_{19} \mathrm{H}_{17} \mathrm{~F}_{3} \mathrm{~N}_{2} \mathrm{O}_{3} \mathrm{~S}$ : C, 55.60; H, 4.18, N, 6.83\%. Found: C, 55.60; H, 4.06, N, 6.62\%.

4-Hydroxy-5-(4-methoxybenzoyl)-6-(4-methoxyphenyl)-4-(trifluoromethyl)tetrahydropyrimidin-2(1H)-thione (4k). White solid in 81\% yield. mp 193-195 ${ }^{\circ} \mathrm{C}$. IR (KBr): $v 3465$ $(-\mathrm{NH}), 3186(-\mathrm{OH}), 2953(\mathrm{ArH}), 1674(\mathrm{C}=\mathrm{O}) \mathrm{cm}^{-1} .{ }^{1} \mathrm{H}$ NMR $\left(400 \mathrm{MHz}, \mathrm{DMSO}-d_{6}\right): \delta 3.68(\mathrm{~s}$, $\left.3 \mathrm{H}, \mathrm{OCH}_{3}\right), 3.79\left(\mathrm{~s}, 3 \mathrm{H}, \mathrm{OCH}_{3}\right), 4.22(\mathrm{~d}, 1 \mathrm{H}, \mathrm{J}=11.3 \mathrm{~Hz}), 5.01(\mathrm{~d}, 1 \mathrm{H}, \mathrm{J}=11.3 \mathrm{~Hz}), 6.70-6.72$ $(\mathrm{dd}, 2 \mathrm{H}, \mathrm{Ar}-\mathrm{H}, \mathrm{J}=6.9 \mathrm{~Hz}), 6.76-6.78(\mathrm{dd}, 2 \mathrm{H}, \mathrm{Ar}-\mathrm{H}, \mathrm{J}=7.0 \mathrm{~Hz}), 7.21-7.23$ (dd, 2H, Ar-Ha-a' $\mathrm{J}=6.7 \mathrm{~Hz}$ ), 7.58-7.60 (dd, 2H, Ar-Hb-b' J = 7.0Hz), $6.94(\mathrm{~s}, 1 \mathrm{H} \mathrm{OH}) 7.70$ (s, 1H, NH), 8.14 (s, $1 \mathrm{H}, \mathrm{NH})$. MS $m / z$ : $440\left(\mathrm{M}^{+}\right)$. Anal. Calcd. for $\mathrm{C}_{20} \mathrm{H}_{19} \mathrm{~F}_{3} \mathrm{~N}_{2} \mathrm{O}_{4} \mathrm{~S}: \mathrm{C}, 54.54 ; \mathrm{H}, 4.35, \mathrm{~N}, 6.36 \%$. Found: C, 54.45; H, 4.26, N, 6.19\%.

4-Hydroxy-5-(4-methoxybenzoyl)-6-(3-methoxyphenyl)-4-(trifluoromethyl)tetrahydropyrimidin-2(1H)-thione (4I). White powder in 82\% yield. mp 245-247 ${ }^{\circ} \mathrm{C}$. IR (KBr): $v 3417$ $(-\mathrm{NH}), 3180(-\mathrm{OH}), 2958(\mathrm{ArH}), 1668(\mathrm{C}=\mathrm{O}) \mathrm{cm}^{-1} .{ }^{1} \mathrm{H}$ NMR $\left(400 \mathrm{MHz}, \mathrm{DMSO}-d_{6}\right): \delta 3.68(\mathrm{~s}$, $\left.3 \mathrm{H}, \mathrm{OCH}_{3}\right), 3.82\left(\mathrm{~s}, 3 \mathrm{H}, \mathrm{OCH}_{3}\right), 4.19(\mathrm{~d}, 1 \mathrm{H}, \mathrm{J}=10.9 \mathrm{~Hz}), 4.96(\mathrm{~d}, 1 \mathrm{H}, \mathrm{J}=11.0 \mathrm{~Hz}), 6.38(\mathrm{~s}, 1 \mathrm{H}$, $\mathrm{NH}), 7.06(\mathrm{~s}, 1 \mathrm{H}, \mathrm{NH}), 6.70-7.59(\mathrm{~m}, 8 \mathrm{H}, \mathrm{Ar}-\mathrm{H})$. MS $m / z: 440\left(\mathrm{M}^{+}\right)$. Anal. Calcd. for $\mathrm{C}_{20} \mathrm{H}_{19} \mathrm{~F}_{3} \mathrm{~N}_{2} \mathrm{O}_{4} \mathrm{~S}: \mathrm{C}, 54.54 ; \mathrm{H}, 4.35$, N, 6.36\%. Found: C, 54.44; H, 4.27, N, 6.21\%.

4-Hydroxy-5-(4-methoxybenzoyl)-6-(2-methoxyphenyl)-4-(trifluoromethyl)tetrahydropyrimidin-2(1H)-thione $(\mathbf{4 m})$. Solid white in $84 \%$ yield. mp 185-187 ${ }^{\circ} \mathrm{C}$. IR $(\mathrm{KBr}): v 3448$ 
$(-\mathrm{NH}), 3192(-\mathrm{OH}), 3232(\mathrm{ArH}), 1670(\mathrm{C}=\mathrm{O}) \mathrm{cm}^{-1} .{ }^{1} \mathrm{H}$ NMR $\left(400 \mathrm{MHz}, \mathrm{DMSO}-d_{6}\right): \delta 3.73$ $\left(\mathrm{s}, 3 \mathrm{H}, \mathrm{OCH}_{3}\right), 3.81\left(\mathrm{~s}, 3 \mathrm{H}, \mathrm{OCH}_{3}\right), 4.56(\mathrm{~d}, 1 \mathrm{H}, \mathrm{J}=11.0 \mathrm{~Hz}), 5.26(\mathrm{~d}, \mathrm{Ha}, \mathrm{J}=11.1 \mathrm{~Hz}), 6.64-7.59$ (m, 10H, Ar-H). MS m/z: $440\left(\mathrm{M}^{+}\right)$. Anal. Calcd. for $\mathrm{C}_{20} \mathrm{H}_{19} \mathrm{~F}_{3} \mathrm{~N}_{2} \mathrm{O}_{4} \mathrm{~S}: \mathrm{C}, 54.54 ; \mathrm{H}, 4.35, \mathrm{~N}$, $6.36 \%$. Found: C, 54.49; H, 4.22, N, 6.17\%.

4-Hydroxy-5-(4-methoxybenzoyl)-6-(4-chlorophenyl)-4-(trifluoromethyl)tetrahydropyrimidin-2(1H)-thione (4n). Solid white in 79\% yield. mp 204-206 ${ }^{\circ} \mathrm{C}$. IR (KBr): v $3483(-\mathrm{NH})$, $3178(-\mathrm{OH}), 2918(\mathrm{ArH}), 1666(\mathrm{C}=\mathrm{O}) \mathrm{cm}^{-1} .{ }^{1} \mathrm{H}$ NMR (400MHz, DMSO-d $): \delta 3.80(\mathrm{~s}, 3 \mathrm{H}$, $\left.\mathrm{OCH}_{3}\right), 4.23(\mathrm{~d}, 1 \mathrm{H}, \mathrm{J}=11.0 \mathrm{~Hz}), 5.19(\mathrm{~d}, 1 \mathrm{H}, \mathrm{J}=11.0 \mathrm{~Hz}), 6.61(\mathrm{~s}, 1 \mathrm{H}, \mathrm{NH}), 6.90(\mathrm{~s}, 1 \mathrm{H}, \mathrm{NH})$, 6.73-8.25 (m, 8H, Ar-H). MS m/z: $444\left(\mathrm{M}^{+}\right)$. Anal. Calcd. for $\mathrm{C}_{19} \mathrm{H}_{16} \mathrm{ClF}_{3} \mathrm{~N}_{2} \mathrm{O}_{3} \mathrm{~S}: \mathrm{C}, 51.30 ; \mathrm{H}$, 3.63 , N, 6.30\%. Found: C, 51.18; H, 3.55, N, 6.17\%.

\section{4-Hydroxy-5-(4-methoxybenzoyl)-6-(4-nitrophenyl)-4-(trifluoromethyl)tetrahydro-}

pyrimidin-2(1H)-thione (4o). Pale yellow powder in $80 \%$ yield. mp 209-211 ${ }^{\circ} \mathrm{C}$. IR (KBr): $v$ $3420(-\mathrm{NH}), 3196(-\mathrm{OH}), 3229,(\mathrm{ArH}), 1676(\mathrm{C}=\mathrm{O}) \mathrm{cm}^{-1} .{ }^{1} \mathrm{H}$ NMR (400MHz, DMSO- $\left.d_{6}\right): \delta 3.82$ $\left(\mathrm{s}, 3 \mathrm{H}, \mathrm{OCH}_{3}\right), 4.15(\mathrm{~d}, 1 \mathrm{H}, \mathrm{J}=11.0 \mathrm{~Hz}), 5.00-5.03(\mathrm{~d}, 1 \mathrm{H}, \mathrm{J}=11.0 \mathrm{~Hz}), 6.84(\mathrm{~s}, 1 \mathrm{H}, \mathrm{NH}), 6.93$ $(\mathrm{s}, 1 \mathrm{H}, \mathrm{NH}), 6.75 .7 .58(\mathrm{~m}, 8 \mathrm{H}, \mathrm{Ar}-\mathrm{H})$. MS m/z: $455\left(\mathrm{M}^{+}\right)$. Anal. Calcd. for $\mathrm{C}_{19} \mathrm{H}_{16} \mathrm{~F}_{3} \mathrm{~N}_{3} \mathrm{O}_{5} \mathrm{~S}: \mathrm{C}$, 50.11; H, 3.54, N, 9.23\%. Found: C, 49.98; H, 3.49, N, 9.12\%.

\section{Acknowledgements}

The authors thank the Department of Chemistry, Saurashtra University, Rajkot for providing facilities. We are also thankful to CIL, RSIC, Chandigarh for providing ${ }^{1} \mathrm{H}$ NMR spectral analysis and SAIF, CDRI Lucknow for elemental analysis of compounds.

\section{References}

1. Xie, W.-H.; Jin, Y.-F.; Wang, P. G. Chemtech 1999, 29, 23.

2. Varma, R. S. Green Chem. 1999, 1, 43.

3. Kidwai M. Pure Appl. Chem. 2001, 73, 147.

4. Kidwai, M.; Sapra, P. Org. Prep. Proced. Int. 2001, 33, 381.

5. Dittmer, D. C. Chem. Ind. 1997, 779.

6. (a) Caddick, S. Tetrahedron 1995, 51, 10403. (b) Kidwai, M.; Venkataramanan, R.; Dave, B. Green Chem. 2001, 3, 278.

7. Kidwai, M.; Venkatramanan, R.; Garg, R. K.; Bhushan, K. R. J. Chem. Res. 2000, 586.

8. (a) Tabern, D. L.; Volwiler, E. H. J. Am. Chem. Soc. 1935, 57, 1963. (b) Atwal, K. S.; Rovnyak, G. C.; O’Reilly, B. C.; Schwartz J. J. Org. Chem. 1989, 54, 5898. (c) Kappe,C. O.; Fabian W. M. F.; Semones, M. A. Tetrahedron 1997, 53, 2803.

9. Biginelli, P. Gazz. Chim. Ital. 1893, 23, 360. 
10. (a) Folkers, K.; Harwood, H. J.; Johnson, T. B. J. Am. Chem. Soc. 1932, 54, 3751. (b) Wipf, P.; Cunningham, A. Tetrahedron Lett. 1995, 36, 7819.

11. (a) Hu, E. H.; Sidler, D. R.; Dolling, U.-H. J. Org. Chem. 1998, 63, 3454. (b) Lu, J.; Ma, H. Synlett 2000, 63. (c) Ranu, B. C.; Hajra, A.; Jana, U. J. Org. Chem. 2000, 65, 6270. (d) Ramalinga, K.; Vijayalakshmi, P.; Kaimal, T. N. B. Synlett 2001, 863. (e) Lu, J.; Bai, Y.; Wang, Z.; Yang, B.; Ma, H. Tetrahedron Lett. 2000, 41, 9075. (f) Yadav, J. S.; Reddy, B. V. S.; Srinivas, R.; Venugopal, C.; Ramalingam, T. Synthesis 2001, 1341. (g) Kumar, K. A.; Kasthuraiah, M.; Reddy, C. S.; Reddy, C. D. Tetrahedron Lett. 2001, 42, 7873. (h) Yadav, J. S.; Reddy, B. V. S.; Reddy, K. B.; Raj, K. S.; Prasad, A. R. J. Chem. Soc., Perkin Trans. 1 2001, 1939. (i) Nandurkar, N. S.; Bhanushali, M. J.; Bhor, M. D.; Bhanage, B. M. J. Mol. Catal. 2007, 271, 14. (j) Shirini, F.; Marjani, K.; Nahzomi, H. T. ARKIVOC 2007, (i), 51. (k) Ma, Y.; Qian, C.; Wang, L.; Yang, M. J. Org. Chem. 2000, 65, 3864-3868. (1) Han, X.; Xu, F.; Luo, Y.; Shen, Q. Eur. J. Org. Chem. 2005, 8, 1500-1504. (m) Legeay, J. C.; Eynde, J. V.; Toupet, L.; Bazureau, J. P. ARKIVOC 2007, (iii), 13.

12. Ranu, B. C.; Hajra, A; Dey, S. S. Organic Process Research \& Development 2002, 6, 817.

13. Kidwai, M.; Saxena, S.; Mohan, R.; Veknkatramanan, R. J. Chem. Soc., Perkin Trans. 1 2002, 1845.

14. (a) Kappe, C. O. 100 Tetrahedron 1993, 49, 6937. (b) Kappe, C. O. Acc. Chem. Res. 2000, 33, 879 .

15. Burgart, Ya. V.; Kuzueva, O. G.; Pryadeina, M. V.; Kappe, C. O.; Saloutin, V. I. Rus. J. Org. Chem. 2001, 37, 869.

16. Saloutin, V. I.; Burgart, Ya. V.; Kuzueva, O. G.; Kappe, C. O.; Chupakhin O. N. J. Fluorine Chem. 2000, 103, 17.

17. Kappe, C. O.; Falsone, S. F. Synlett 1998, 718.

18. Bose, S. D.; Sudharshan, M.; Chavhan S. W. ARKIVOC 2005, (iii), 228. 